\title{
Clinical Vignettes \\ Expanding the differential diagnosis for transaminitis in patients with anorexia nervosa
}

\author{
Adam C. Nadelson, M.D. ${ }^{7}$, Victor D. Babatunde, B.S. ${ }^{7}$, Eric U. Yee, M.D. ${ }^{2}$, and Vilas R. Patwardhan, \\ $M . D .^{3}$ \\ 'Department of Medicine, Beth Israel Deaconess Medical Center, Harvard Medical School, Boston, MA, USA; ${ }^{2}$ Department of Pathology, Beth \\ Israel Deaconess Medical Center, Harvard Medical School, Boston, MA, USA; ${ }^{3}$ Department of Medicine, Liver Center, Beth Israel Deaconess \\ Medical Center, Harvard Medical School, Boston, MA, USA.
}

Aminotransferase elevations have been described in patients with anorexia nervosa. Hypothesized etiologies have included ischemic hepatitis, refeeding-induced transaminitis, and the process of autophagy. Supervised enteral nutrition is the mainstay of treatment for severe anorexia, but an increase in aminotransferase levels after initiation of enteral feeding presents clinicians with a diagnostic dilemma. We present a 31-year-old woman with anorexia nervosa (body mass index [BMI] of $13.5 \mathrm{~kg} / \mathrm{m}^{2}$ ) who experienced a worsening of aminotransferase elevations even after the initiation of enteral feeding. Despite nutritional supplementation, the patient's weight continued to fall for 6 days. Peak aminotransferase concentrations correlated with the patient's lowest weight and improved only after an increase in BMI was eventually achieved. Secondary causes of severe transaminitis were investigated, and after no cause was found, a liver biopsy was performed. Pathology was consistent with liver injury secondary to severe malnutrition rather than from refeeding syndrome. This case highlights malnutrition as an important cause of aminotransferase elevations and underscores the need for judicious early weight restoration in patients with anorexia and abnormal liver chemistry.

KEY WORDS: clinical vignette; hepatitis; hospital medicine; anorexia.

Abbreviations

ALT Alanine aminotransferase

Anti-TTG-

IgA Anti-tissue transglutaminase-immunoglobulin A

AST Aspartate aminotransferase

BMI Body mass index

IU/L International units per liter

$\mathrm{kg} \quad$ Kilograms

$\mathrm{kg} / \mathrm{m} 2 \quad$ Kilogram per meter squared

J Gen Intern Med 32(4):486-9

DOI: $10.1007 / \mathrm{s} 11606-016-3879-8$

(c) Society of General Internal Medicine 2016

\section{CLINICAL VIGNETTE}

A 31-year-old woman with anxiety and a 3-year history of restricting-subtype anorexia nervosa was referred to our hospital for evaluation of elevated aminotransferase levels, which were discovered during a routine outpatient medical evaluation.

Published online October 31, 2016
On admission, alanine aminotransferase (ALT) was $514 \mathrm{IU} /$ L (reference range: 0-40 IU/L), aspartate aminotransferase (AST) was $130 \mathrm{IU} / \mathrm{L}$ (reference range: 0-40 IU/L), and alkaline phosphatase and total bilirubin levels were normal. Her past aminotransferase levels were not available from previous outpatient providers. The patient was asymptomatic without fevers, chills, night sweats, nausea, vomiting, or abdominal pain. She did acknowledge having loose stools after consuming gluten-containing products. She denied any change in the color of her stools or urine. She denied recent alcohol consumption, use of acetaminophen or herbal supplements, highrisk sexual behavior, or intravenous drug use. She did have a history of heavy alcohol consumption (approximately 10 drinks per day) for a 10-year period, but had quit 3 years prior to presentation. She had a history of smoking tobacco but also quit 3 years prior to presentation. She had used cocaine at the age of 19 but denied any recreational drug use since that time. Outpatient medications included a daily multivitamin and $1000 \mathrm{mg} /$ day of ascorbic acid. She had no allergies to medications. There was no family history of liver disease.

Vital signs on admission were notable for a blood pressure of 93/62 mmHg and heart rate of 56 beats per minute. Admission weight was $35.7 \mathrm{~kg}$ (BMI: $13.5 \mathrm{~kg} / \mathrm{m}^{2}$ ). She was thin appearing, with anicteric sclera. She had lanugo superior to the upper lip, and along her extremities and torso. Cardiovascular examination was notable for bradycardia. Lungs were unremarkable. A scaphoid abdomen with loss of subcutaneous fat was appreciated but was soft, non-tender, and without rebound or guarding. There was no hepatosplenomegaly. Skin did not show evidence of jaundice, petechiae, ecchymoses, spider angioma, palmar erythema, caput medusa, or cyanosis. Extremities revealed loss of muscle mass in the calves, but no edema. There was no asterixis or altered mental status.

Admission laboratory evaluation revealed a normal complete blood count. Chemistry panel was normal except for plasma glucose of $68 \mathrm{mg} / \mathrm{dL}$, sodium $130 \mathrm{mEq} / \mathrm{L}$, chloride $94 \mathrm{mEq} / \mathrm{L}$, and bicarbonate $31 \mathrm{mEq} / \mathrm{L}$. Alkaline phosphatase was $63 \mathrm{IU} / \mathrm{L}$, with total bilirubin of $0.6 \mathrm{mg} / \mathrm{dL}$ and albumin $4.9 \mathrm{~g} / \mathrm{dL}$. Coagulation studies were normal. An evaluation for acute causes of elevated aminotransferases was negative (Table 1), including acute hepatitis A, acute hepatitis B, acute hepatitis C, cytomegalovirus, and Epstein-Barr virus. Serum toxicology screen, 
Table 1 Acute and Chronic Causes of Elevated Aminotransferases Considered in Our Patient

\begin{tabular}{ll}
\hline \hline Causes of acute transaminitis & Causes of chronic transaminitis \\
\hline Acute hepatitis A & $\begin{array}{l}\text { Chronic hepatitis B and chronic } \\
\text { hepatitis C }\end{array}$ \\
Acute hepatitis B & Iron overload syndromes \\
Acute hepatitis C & Autoimmune liver disease* \\
Cytomegalovirus & Thyroid disorders \\
Epstein-Barr Virus & Wilson's disease \\
Toxic ingestions (acetaminophen, & Celiac disease \\
alcohol) & \\
\hline
\end{tabular}

*Autoimmune liver disease $=$ autoimmune hepatitis (AIH), primary biliary cholangitis (PBC), and primary sclerosing cholangitis (PSC)

including acetaminophen, was negative. Urine toxicology screen and an ethanol level were not obtained on admission.

An evaluation for chronic causes of elevated aminotransferases was undertaken and was negative for chronic hepatitis $\mathrm{B}$ and $\mathrm{C}$, iron overload syndromes, autoimmune liver disease, thyroid disorders, and Wilson's disease. Anti-tissue transglutaminase-IgA (anti-TTG-IgA) for celiac disease was elevated at greater than 100 units. An abdominal ultrasound showed normal hepatic parenchyma with a smooth liver contour and patent portal vein, without gallbladder or biliary duct abnormalities.

At this point in the patient's course, the hepatology service was consulted, as it was unclear whether celiac disease, anorexia, or another etiology was responsible for the elevated aminotransferases. The medical team felt that she required nutritional support for treatment of her anorexia, and the patient was started on a multi-disciplinary eating disorder protocol, with daily weight and strict meal requirements. She was fed a gluten-free diet in view of the elevated anti-TTGIgA and probable celiac disease.

After the initiation of supplemental nutrition, aminotransferases worsened, reaching their peak on hospital day 6 , with an ALT $1368 \mathrm{IU} / \mathrm{L}$ and AST $544 \mathrm{IU} / \mathrm{L}$, raising concern for refeeding-induced hepatitis. There was no clinical evidence of refeeding syndrome, however, and serum electrolytes remained stable, without requiring repletion. A liver biopsy was performed on this day and revealed relatively minimal changes, which appeared to be discrepant from the severely elevated aminotransferase levels (Fig. 1). Focal portal areas contained mild mononuclear inflammation, while the lobules showed no significant inflammation and only rare acidophil bodies (apoptotic hepatocytes). Centrivenular zones showed some sinusoidal dilatation with hepatocytes that appeared slightly atrophic. There was no steatosis, ballooning degeneration, or Mallory hyaline. There was also no lymphocytic proliferation. The disparity between minimal histologic changes by light microscopy and markedly elevated serum aminotransferases was felt to be consistent with severe malnutrition and not refeeding syndrome, alcoholic liver disease, or celiac disease.

Daily patient weight and hepatic laboratory values are summarized in Table 2. As noted, peak aminotransferase levels

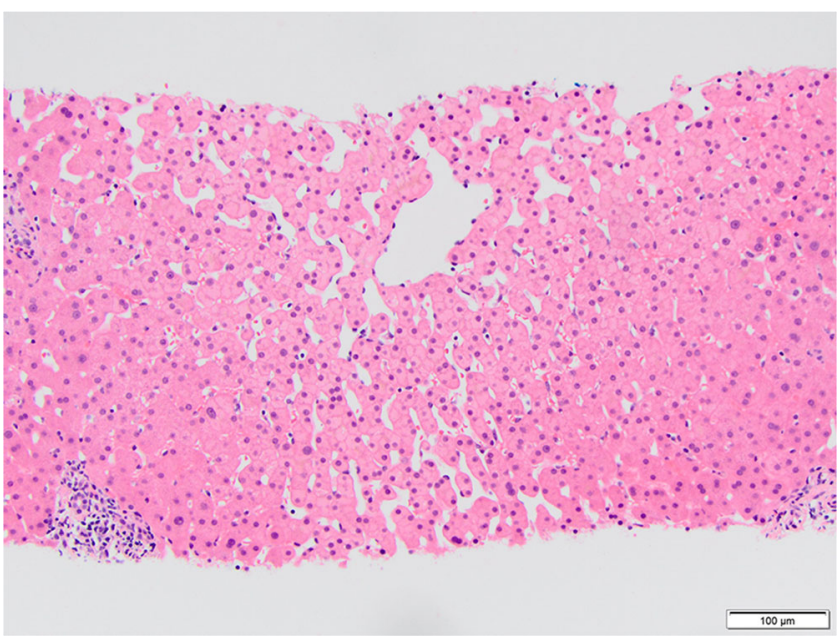

Figure 1 Liver biopsy. (H\&E $\times \mathbf{2 0 0}$ magnification). One portal tract shows mild mononuclear inflammation without interface or lobular activity (bottom left). The centrivenular zone shows sinusoidal dilatation and some hepatocytes that appear slightly atrophic (center).

corresponded to a nadir in weight at the time of liver biopsy, also suggesting malnutrition-induced transaminitis. Supplemental nutrition was continued, and with steady weight gain, aminotransferase levels trended downward, with an ALT of $100 \mathrm{IU} / \mathrm{L}$ and AST of $47 \mathrm{IU} / \mathrm{L}$ at a peak weight of $52.2 \mathrm{~kg}$ (BMI $\left.19.8 \mathrm{~kg} / \mathrm{m}^{2}\right), 84$ days after her initial presentation.

In summary, this patient's presentation was consistent with transaminitis secondary to severe malnutrition, and not refeeding syndrome, in a patient with underlying untreated celiac disease (Table 3 ).

Table 2 Daily weight and liver chemistry values from the time of initial hospital presentation

\begin{tabular}{lllll}
\hline $\begin{array}{l}\text { Days since initial } \\
\text { presentation }\end{array}$ & $\begin{array}{l}\text { Weight } \\
\mathbf{( k g )}\end{array}$ & $\begin{array}{l}\text { Body mass } \\
\text { index } \\
\mathbf{m}^{\mathbf{2}} \text { ) }\end{array}$ & $\begin{array}{l}\text { ALT } \\
\text { (IU/L) }\end{array}$ & $\begin{array}{l}\text { AST } \\
\text { (IU/L) }\end{array}$ \\
\hline 0 & - & - & 514 & 130 \\
1 & 35.7 & 13.5 & 462 & 146 \\
2 & 34.7 & 13.1 & 554 & 160 \\
3 & 34.8 & 13.2 & 723 & 305 \\
4 & 34.6 & 13.1 & 976 & 455 \\
5 & 34.0 & 12.9 & 1133 & 403 \\
6 & 33.7 & 12.8 & 1368 & 544 \\
7 & 33.6 & 12.7 & 1262 & 463 \\
8 & 33.7 & 12.8 & 1115 & 322 \\
9 & 34.1 & 12.9 & - & - \\
10 & 35.1 & 13.3 & 1054 & 347 \\
11 & 35.1 & 13.3 & 816 & 149 \\
12 & 35.0 & 13.2 & 741 & 157 \\
13 & 35.0 & 13.2 & 528 & 94 \\
14 & 35.8 & 13.5 & 525 & 114 \\
40 & 38.0 & 14.4 & 110 & 58 \\
48 & 38.4 & 14.5 & 142 & 63 \\
84 & 52.2 & 19.8 & 100 & 47 \\
\hline
\end{tabular}




\section{Table 3 Key Learning Points}

1. Differentiating transaminitis secondary to severe malnutrition from transaminitis secondary to refeeding syndrome can pose a diagnostic

dilemma. The distinction is important, since the treatment of the former is to encourage enteral feeding, whereas the treatment of the latter is to withhold enteral nutrition.

2. Abnormal aminotransferases are a marker of disease severity in patients with anorexia nervosa.

3. Abnormal aminotransferases in anorexia may be a consequence of autophagy.

\section{DISCUSSION}

This case demonstrates severe malnutrition (defined as energy intake $\leq 50 \%$ energy intake compared to estimated energy needs for $\geq 1$ month, severe body fat depletion, severe muscle mass depletion) to be an important cause of elevated aminotransferase levels, and highlights abnormal liver chemistry as a sign of clinically severe malnutrition.

The diagnostic dilemma that arises with initiation of enteral nutrition when caring for patients with low BMI and abnormal liver chemistry is the risk of refeeding syndrome with continued enteral nutrition, versus the risk of continued malnutrition with cessation of enteral feeding. Although hepatic steatosis secondary to refeeding has been implicated in elevated ALT and AST in anorexia nervosa patients with malnutrition, ${ }^{2}$ there was no evidence of hepatic steatosis on our patient's liver biopsy specimen. Additionally, aminotransferase elevations in refeeding syndrome tends to be lower than aminotransferase elevations secondary to severe malnutrition, with ALT ranging from $193 \mathrm{IU} / \mathrm{L}$ to $218 \mathrm{IU} / \mathrm{L}$ and AST ranging from $157 \mathrm{IU} / \mathrm{L}$ to $173 \mathrm{IU} / \mathrm{L}$, in case reports of transaminitis secondary to refeeding syndrome. ${ }^{3,4}$ While refeeding syndromes are often associated with weight gain, our case demonstrates that patients with severe malnutrition may continue to lose weight even after supervised feedings have begun, placing them at continued risk for rising aminotransferases, liver failure, and complications of anorexia nervosa. In our patient, continued feeding with controlled enteral supplementation eventually led to a decline in transaminases as her weight increased. This is consistent with reports that have shown near normalization of liver enzymes with associated weight gain. ${ }^{5,6}$

Studies have shown a prevalence of aminotransferase elevations of $43-52 \%$ in anorexia nervosa patients on hospital admission. ${ }^{7,8}$ Proposed mechanisms include oxidant stress from low glutathione levels, ischemic hepatitis from low blood flow states, and starvation-induced autophagy., ${ }^{2,7-14}$

Starvation-induced autophagy, where the nutritional stress of malnourishment induces mechanisms of autophagy without evoking an overt process of apoptosis, has been described. In a series of 12 patients with anorexia nervosa (average BMI $11.3 \mathrm{~kg} / \mathrm{m}^{2}$ ) and severe liver injury (average ALT 1,904 IU/ L), Rautou et al. showed the presence of autophagosomes on liver biopsy, with no marked morphological features of necrosis or apoptosis. The group proposed that starvation-induced autophagy led to an increase in the permeability of the hepatocyte plasma membrane and subsequent release of aminotransferases into the blood, even without cell death. ${ }^{9}$ Kheloufi et al. proposed that in anorexic patients with malnutrition, the initial response of autophagy to cope with nutrient depletion is likely a hepatoprotective response by preventing hepatocyte necrosis and hepatic insufficiency. When starvation continues and BMI reaches a dangerous level of $13 \mathrm{~kg} / \mathrm{m}^{2}$ or less, excessive autophagy may no longer be protective, leading to hepatocellular death and liver failure. ${ }^{15}$ Thus, starvation-induced autophagy and hepatocyte plasma membrane permeability can explain the increase in serum aminotransferases in our patient, and absence of hepatocyte necrosis or inflammation on our patient's histology.

Of note, our patient's BMI remained near the $13 \mathrm{~kg} / \mathrm{m}^{2}$ (nadir BMI $12.7 \mathrm{~kg} / \mathrm{m}^{2}$ ), at which point autophagy may have given way to hepatocyte death. Her liver biopsy, showing only rare lobular apoptotic hepatocytes and absence of extensive liver cell necrosis, supports the findings of Kheloufi et al. of a "critical" BMI up to which autophagy can be hepatoprotective. Maintaining her weight above a BMI of $13 \mathrm{~kg} / \mathrm{m}^{2}$ may have prevented further hepatocellular damage and averted hepatic failure.

Table 2 indicates that her nadir weight corresponded to peak aminotransferase levels. Interestingly, aminotransferase levels improved and reached a nadir even with an increase in BMI to just $14.5 \mathrm{~kg} / \mathrm{m}^{2}$, suggesting that there is a BMI at which hepatic autophagy from malnutrition is initiated. Previous studies have shown a similar trend of inverse correlation between the severity of transaminitis and BMI..$^{5-7}$ These findings suggest that aminotransferase levels in patients with severe malnutrition may serve as a marker of disease severity and may thus help to guide monitoring and treatment. ${ }^{16}$

Of note, our patient did have a history of gluten sensitivity and was diagnosed with celiac disease, on the basis of her elevated anti-TTG-IgA, during her hospitalization. Celiac disease has been associated with mild transaminitis, but not to the severity seen here in our case. Bardella et al. evaluated patients with chronically elevated aminotransferases who were found to have celiac disease, with ALT ranging from $48 \mathrm{IU} / \mathrm{L}$ to $130 \mathrm{IU} / \mathrm{L}$, and AST ranging from $29 \mathrm{IU} / \mathrm{L}$ to $80 \mathrm{IU} / \mathrm{L} .{ }^{17}$ Although our patient's diet was changed to a gluten-free diet in view of the elevated anti-TTG-IgA, complete aminotransferase normalization can take up to 1 year following cessation of gluten intake. ${ }^{18}$

We suspect that our patient's continued transaminitis at levels consistent with the findings of Bardella et al., even after improvement of her BMI to $19.8 \mathrm{~kg} / \mathrm{m}^{2}$, may be the result of her underlying celiac disease. Although celiac disease could have led to a baseline elevation in aminotransferases, the degree of our patient's aminotransferase elevation at presentation, and a liver biopsy showing no evidence of the lymphocytic infiltration that would be present with liver damage secondary to celiac disease, is inconsistent with a presentation of celiac disease as the cause of her acute hepatitis. ${ }^{19}$ Additionally, while our patient had a history of heavy alcohol 
consumption, the lack of biopsy findings consistent with alcoholic liver disease, including steatosis, ballooning degeneration, and Mallory hyaline, decreases the likelihood that her mild chronic aminotransferase elevations were secondary to alcoholic liver disease. ${ }^{20}$ It is currently unclear whether celiac disease predisposes anorexic patients to more severe liver complications. However, celiac disease can complicate the provision of enteral nutrition if a gluten-free diet is followed, as such a diet limits enteral nutrition options.

In conclusion, abnormal and severe aminotransferase elevations may be encountered in severe malnutrition and may be a marker of disease severity. Aminotransferase elevations may be encountered even after initiation of refeeding if the BMI does not rise. Differentiating liver dysfunction secondary to severe malnutrition from refeeding syndrome is pivotal, and attention should be paid to the BMI during the course of refeeding, as judicious early weight restoration ought to be pursued, not delayed, for these severely undernourished patients.

Contributors: There were no further contributors to the paper, as the contributors all met criteria for authorship.

Corresponding Author: Adam C. Nadelson, M.D.; Department of Medicine, Beth Israel Deaconess Medical CenterHarvard Medical School, Boston, MA, USA (e-mail: anadelso@bidmc.harvard.edu). Compliance with Ethical Standards:

Conflict of Interest: The authors declare that they have no conflict of interest.

Funders: There was no financial support received for the work presented in the manuscript.

Prior Presentations: The manuscript was presented as a poster titled, "Broadening the Differential for Severe Hepatitis in Anorexia Nervosa," at the Society of General Internal Medicine Annual Meeting in Hollywood, FL, in May 2016.

\section{REFERENCES}

1. White JV, Guenter P, Jensen G, Malone A, Schofield M. Consensus Statement of the Academy of Nutrition and Dietetics/American Society for Parenteral and Enteral Nutrition: characteristics recommended for the identification and documentation of adult malnutrition (Undernutrition). J Acad Nutr Diet. 2012;112(5):730-738.
2. Harris RH, Sasson G, Mehler PS. Elevation of liver function tests in severe anorexia nervosa. Int J Eat Disord. 2013;46(4):369-374.

3. Vootla VR, Daniel M. Abnormal liver function tests in an anorexia nervosa patient and an atypical manifestation of refeeding syndrome. Case Rep Gastroenterol. 2015;9(2):261-265.

4. Korbonits M, Blaine D, Elia M, Powell-Tuck J. Metabolic and hormonal changes during the refeeding period of prolonged fasting. Eur J Endocrinol. 2007; 157(2): 157-166.

5. Narayanan V, Gaudiani JL, Harris RH, Mehler PS. Liver function test abnormalities in anorexia nervosa-cause or effect. Int $\mathrm{J}$ Eat Disord. 2010;43(4):378-381.

6. Rosen E, Sabel AL, Brinton JT, Catanach B, Gaudiani JL, Mehler PS. Liver dysfunction in patients with severe anorexia nervosa. Int $\mathrm{J}$ Eat Disord. 2016;49(2):153-160.

7. Hanachi M, Melchior JC, Crenn P. Hypertransaminasemia in severely malnourished adult anorexia nervosa patients: risk factors and evolution under enteral nutrition. Clin Nutr. 2013;32(3):391-395.

8. Tsukamoto M, Tanaka A, Arai M, et al. Hepatocellular injuries observed in patients with an eating disorder prior to nutritional treatment. Intern Med. 2008;47(16): 1447-1450.

9. Rautou P, Cazals-Hatem D, Moreau R, et al. Acute liver cell damage in patients with anorexia nervosa: a possible role of starvation-induced hepatocyte autophagy. Gastroenterology. 2008;135(3):840-848.

10. Di Pascoli L, Lion A, Milazzo D, Caregaro L. Acute liver damage in anorexia nervosa. Int J Eat Disord. 2004;36(1):114-117.

11. De Caprio C, Alfano A, Senatore I, Zarrella L, Pasanisi F, Contaldo F. Severe acute liver damage in anorexia nervosa: two case reports. Nutrition. 2006;22(5):572-575.

12. Giordano F, Arnone S, Santeusanio F, Pampanelli S. Brief elevation of hepatic enzymes due to liver ischemia in anorexia nervosa. Eat Weight Disord. 2010;15(4):294-297.

13. Dowman J, Ramakrishnan A, Chesner I. Recurrent acute hepatic dysfunction in severe anorexia nervosa. Int J Eat Disord. 2010;43:770772 .

14. Kheloufi M, Boulanger CM, Codogno P, Rautou P. Autosis occurs in the liver of patients with severe anorexia nervosa. Hepatology. 2015;62(2):657658.

15. Kheloufi M, Boulanger CM, Durand F, Rautou PE. Liver autophagy in anorexia nervosa and acute liver injury. Biomed Res Int. 2014;2014:701064

16. Lelli L, Castellini G, Gabbani T, Godini L, Rotella F, Ricca V. Associations between liver enzymes, psychopathological and clinical features in eating disorders. Eur Eat Disord Rev. 2014;22(6):443-447.

17. Bardella MT, Vecchi M, Conte D, et al. Chronic unexplained hypertransaminasemia may be caused by occult celiac disease. Hepatology. 1999;29(3):654-657.

18. Sainsbury A, Sanders DS, Ford AC. Meta-analysis: Coeliac disease and hypertransaminasaemia. Aliment Pharmacol Ther. 2011;34(1):33-40.

19. Mounajjed T, Oxentenko A, Shmidt E, Smyrk T. The liver in celiac disease: clinical manifestations, histologic features, and response to gluten-free diet in 30 patients. Am J Clin Pathol. 2011;136:128-137.

20. Gao B, Bataller R. Alcoholic liver disease: pathogenesis and new therapeutic targets. Gastroenterology. 2011;141:1572-1585. 